\title{
Colloidal Synthesis of Laterally Confined Blue-Emitting 3.5 Monolayer CdSe Nanoplatelets
}

\author{
Alessio Di Giacomo, Carmelita Rodà, Ali Hossain Khan, and Iwan Moreels* \\ Cite This: Chem. Mater. 2020, 32, 9260-9267 \\ Read Online
}

ABSTRACT: The typical synthesis protocol for blue-emitting CdSe nanoplatelets (NPLs) yields particles with extended lateral dimensions and large surface areas, resulting in NPLs with poor photoluminescence quantum efficiency. We have developed a synthesis protocol that achieves an improved control over the lateral size, by exploiting a series of long-chained carboxylate precursors that vary from cadmium octanoate $\left(C_{8}\right)$ to cadmium stearate $\left(C_{18}\right)$. The length of this metallic precursor is key to tune the width and aspect ratio of the final NPLs, and for the shorter chain lengths, the synthesis yield is improved. NPLs prepared with our procedure possess significantly

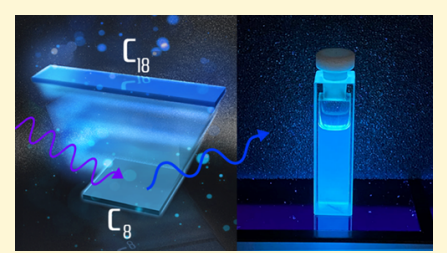
enhanced photoluminescence quantum efficiencies, up to $30 \%$. This is likely due to their reduced lateral dimensions, which also grant them good colloidal stability. As the NPL width can be tuned below the bulk exciton Bohr radius, the band edge blue-shifts, and we constructed a sizing curve relating the NPL absorption position and width. Further adjusting the synthesis protocol, we were able to obtain even thinner NPLs, emitting in the near-UV region, with a band-edge quantum efficiency of up to $11 \%$. Results pave the way to stable and efficient light sources for applications such as blue and UV light-emitting devices and lasers.

\section{INTRODUCTION}

Since the discovery of wurtzite $^{1}$ and zincblende $^{2} \mathrm{CdSe}$ nanoplatelets (NPLs) in 2006 and 2008, respectively, colloidal two-dimensional (2D) semiconductor NPLs have established themselves as a novel and exciting class of materials. The onedimensional (1D) quantum confinement, acting only along the thickness direction, labels them as a colloidal counterpart of quantum wells, ${ }^{3,4}$ and is the pivotal element for their peculiar optoelectronic properties. In comparison to zero-dimensional (OD) colloidal quantum dots, they exhibit narrower emission line widths, faster photoluminescence (PL) decay times, ${ }^{5}$ suppressed Auger recombination ${ }^{6}$ so that they achieve high optical gain coefficients, ${ }^{6-8}$ and a large nonlinear absorption coefficient. $^{9}$ They yield bright and pure-color light-emitting diodes, ${ }^{10,11}$ and their geometry makes them potential candidates for chemical sensing ${ }^{12}$ thanks to the high surfaceto-volume ratio. Established procedures, mostly based on metal carboxylates as precursors, are available to prepare highly luminescent zincblende CdSe NPLs with atomically precise control over their thickness. ${ }^{3,13-15}$ The latter, verifiable by absorption and photoluminescence (PL) spectroscopy, is usually defined by the number $N$ of monolayers (ML), with $N$ being the number of Se crystalline planes bound to the $N+$ 1 planes of Cd. NPLs already have shown emission over the visible and UV spectral range, from $395 \mathrm{~nm}$, for 2.5 ML NPLs, to $625 \mathrm{~nm}$, for $8.5 \mathrm{ML}$ NPLs, ${ }^{3,13,16}$ yet most efforts are directed toward highly fluorescent 4.5 ML and 5.5 ML NPLs, emitting around 510 and $550 \mathrm{~nm}$, respectively.

In this work, we focus on the thinner NPLs, ${ }^{17}$ emitting in the blue region of the electromagnetic spectrum $\left(2.7 \mathrm{eV}=E_{\mathrm{g}}, \lambda\right.$ $=460 \mathrm{~nm}$ ). Reducing the NPL thickness is expected to influence the linear and nonlinear optical properties. While

there are reports for the preparation of $3.5 \mathrm{ML} \mathrm{CdSe}$ NPLs, ${ }^{3,18-20}$ in most, the well-established preparation of 4.5 ML NPLs was essentially modified by lowering the NPL growth temperature. ${ }^{3,18,21}$ This approach yields extended nanosheets that are not ideally suited for further investigation of the NPL photophysics or final optoelectronic or photonic applications, as the NPLs display enhanced Rayleigh scattering and carrier trapping at surface defects. Therefore, it is paramount to achieve a lateral size tuning, without relying critically on the temperature to obtain thinner NPLs.

We resolved this issue by unraveling the effect of the organic chain length of the initial cadmium carboxylate on the precursor conversion rate and final lateral dimensions of the CdSe NPLs. We demonstrate that it is possible to tune the overall area and aspect ratio of such NPLs using different chain lengths. We then investigated the optical properties of the synthesized NPLs by means of absorbance spectroscopy, and steady-state and time-resolved photoluminescence (PL) spectroscopy. The NPLs show narrow-band PL spectra ( $8 \mathrm{~nm}$ full width at half-maximum, FWHM) with a low contribution of trap emission. The band-edge PL quantum efficiency (PL QE) of $5-30 \%$ exceeds recent literature confirming the reduced influence of surface trapping. ${ }^{3,18,21,22}$ A fast (4-8 ns) fluorescence lifetime is in line with the enhanced absorption

Received: July 24, 2020

Revised: October 9, 2020

Published: October 21, 2020 

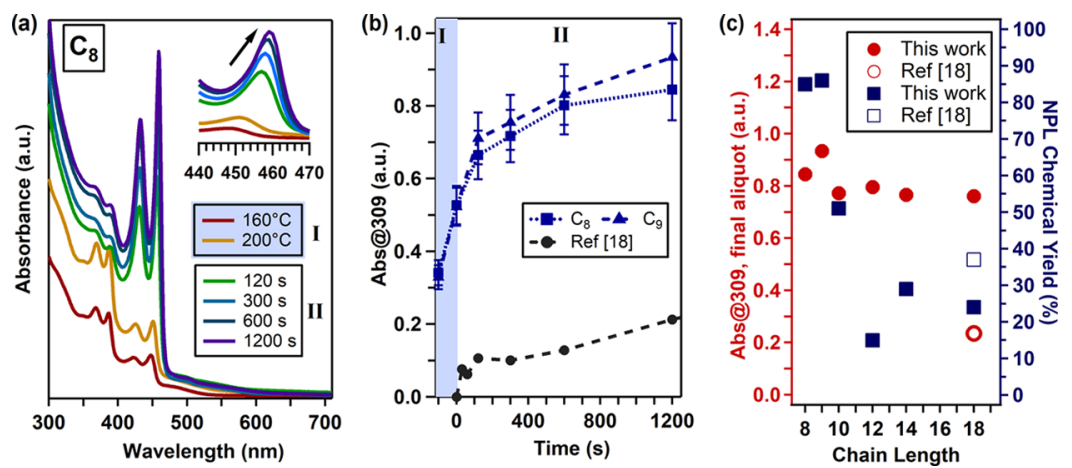

Figure 1. (a) Temporal evolution of the absorbance spectra for aliquots collected during a typical synthesis (cadmium octanoate, $\mathrm{C}_{8}$ ). Regions $\mathrm{I}$ and II: time before and after propionic acid injection, respectively. Inset: intensity and spectral shift of the e-hh transition. (b) Absorbance values at $309 \mathrm{~nm}$ for $\mathrm{C}_{8}$ and $\mathrm{C}_{9}$ carboxylate chain lengths (blue), compared to a reference procedure (black). ${ }^{18}$ Regions I and II: before and after propionic acid injection, respectively. A higher conversion into CdSe is observed, attributed to the higher synthesis temperature (error bars, $\pm 10 \%$ of the absorbance value). (c) Left axis: Abs@309 for the final aliquots prepared using procedure (1) (filled red circles), and using ref 18 (empty red circle). Right axis: chemical yield in 3.5 ML CdSe NPLs, as a function of the chain length of the initial cadmium carboxylate for procedure (1) (filled blue squares), and using ref 18 (empty blue square).

coefficient compared to 4.5 ML NPLs, yet can be attributed to the reduced NPL thickness and blue-shifted band gap, rather than an enhanced band-edge oscillator strength. Finally, by slightly adjusting the synthesis conditions, we also managed to isolate $2.5 \mathrm{ML}$ CdSe NPLs, which are characterized by even shorter lifetimes of $3 \mathrm{~ns}$, and, considering the $0.76 \mathrm{~nm}$ thickness, an appreciable band-edge PL QE of 5-11\%.

\section{RESULTS AND DISCUSSION}

The synthetic investigation is oriented toward control over area and lateral aspect ratio (AR) of $3.5 \mathrm{ML}$ CdSe NPLs. While a modification of lateral dimensions can be obtained by simply arresting the reaction at earlier times, ${ }^{23}$ this approach is not particularly convenient considering the lower chemical yield. Therefore, we investigated the effect of the cadmium carboxylate precursor on the lateral sizes of 3.5 ML NPLs. Using different chain lengths $\left(\mathrm{C}_{8}-\mathrm{C}_{18}\right)$, our synthesis method is summarized in eq 1

$$
\mathrm{Cd}\left(\mathrm{O}_{2} \mathrm{CR}\right)_{2}+\mathrm{Se}-\mathrm{ODE} \underset{(2)}{\stackrel{(1)}{\rightarrow}} 3.5 \mathrm{ML} \text { CdSe NPLs }
$$

(1) $=200{ }^{\circ} \mathrm{C}, \mathrm{CH}_{3} \mathrm{CH}_{2} \mathrm{COOH}$. (2) $220{ }^{\circ} \mathrm{C}, 20 \mathrm{~min}(\mathrm{R}=$ $\left.-\mathrm{C}_{7} \mathrm{H}_{15},-\mathrm{C}_{8} \mathrm{H}_{17},-\mathrm{C}_{9} \mathrm{H}_{19},-\mathrm{C}_{11} \mathrm{H}_{23}-\mathrm{C}_{13} \mathrm{H}_{27}, \mathrm{C}_{17} \mathrm{H}_{35}\right)$

Step (1) indicates the temperature at which we inject propionic acid, and step (2) indicates the final temperature and growth time. The advantages of our approach are threefold. First, it involves a higher reaction temperature with respect to the reference procedure $\left(220\right.$ vs $\left.170{ }^{\circ} \mathrm{C}\right),{ }^{18}$ which is expected to benefit nucleation of NPL seeds. ${ }^{24-26}$ Second, initial experiments evidenced the possibility to obtain NPLs by injecting a short-chain carboxylic acid, avoiding the addition of secondary solid cadmium salt precursor. $2,15,23,27,28$ Concomitantly, the amount of unreacted cadmium is significantly reduced. Finally, the injection of a liquid, compared to a solid powder, is typically more controllable. Based on initial investigations, we selected propionic acid as short-chain ligand.

The typical time evolution of the absorbance spectra obtained by procedure (1), using cadmium octanoate $\left(\mathrm{C}_{8}\right)$, is shown in Figure 1a. Profiles for the other carboxylates are similar (Supporting Information Figure S1). The time spans before and after injection of propionic acid are defined as region I (blue shaded) and II, respectively. Clearly, already before the addition of the short-chain carboxylic acid, signals attributable to excitonic features (e-lh and e-hh) of small 2.5 ML (around 368 and $387 \mathrm{mn}$ ) and $3.5 \mathrm{ML}$ (422 and $448 \mathrm{~nm}$ ) CdSe NPLs appear in the absorbance spectra of the aliquots. Transmission electron microscopy (TEM) analysis of these aliquots (SI, Figure S2) corroborates this, showing the presence of small NPLs already at this stage, in accordance with recent small-angle X-ray scattering (SAXS) measurements ${ }^{29}$ and studies on the kinetic instability of NPLs. ${ }^{30}$ Note that we also observed that the presence of propionic acid is not necessary to obtain NPLs under kinetic growth conditions, ${ }^{20,31}$ yet it allows us to obtain a more homogeneous result, with less byproducts (SI, Figure S3). The inset in Figure 1a confirms that our procedure allows for lateral size control, as the bandedge absorption evolves from $448 \mathrm{~nm}$ before propionic acid addition, to $460 \mathrm{~nm}$ after a $1200 \mathrm{~s}$ growth at $220{ }^{\circ} \mathrm{C}$. To investigate the hypothesis of an enhanced nucleation rate with our method, we took aliquots (always of the same volume) during the growth and measured the relative amount of $\mathrm{CdSe}$ produced by their absorbance at 309 nm (Abs@309, assuming that this value does not change significantly with the NPL dimensions ${ }^{9}$ ), as shown in Figure $1 \mathrm{~b}$. As a reference, we compared our data with $3.5 \mathrm{ML}$ CdSe NPLs synthesized according to the literature protocol of Ithurria et al. ${ }^{18}$ Note that, compared to literature, we have increased the molar amount of selenium (the limiting reagent) during synthesis, from $0.15^{18}$ to $0.25 \mathrm{mmol}$. In the figure, regions I (blue) and II again mark the time before and after propionic acid injection, respectively. As Abs@309 is proportional to the amount of crystalline CdSe produced during the reaction, ${ }^{9}$ Figure $1 \mathrm{~b}$ demonstrates a higher conversion of precursors using the current method with $\mathrm{C}_{8}$ ligands, which is corroborated using $\mathrm{C}_{9}$ ligands. Using longer chain lengths $\left(\mathrm{C}_{10}-\mathrm{C}_{18}\right)$, however, we observed that the reaction starts to proceed via two pathways, yielding both NPLs and a sizable fraction of QDs ${ }^{20}$ (Figure S1 shows the absorbance spectra). The overall amount of CdSe remained high (Figure 1c, red circles), in line with previous studies on the synthesis of CdSe QDs demonstrating higher nanocrystal conversion using Se-ODE, compared to TOP-Se as in the reference procedure, ${ }^{18,32}$ yet we obtained an increasing contribution of QDs as the chain length increases. The results therefore indicate that short-chain ligands are beneficial to maintain a kinetic growth regime and avoid undesired isotropic QD growth. To extract the actual NPL yield, we corrected our 

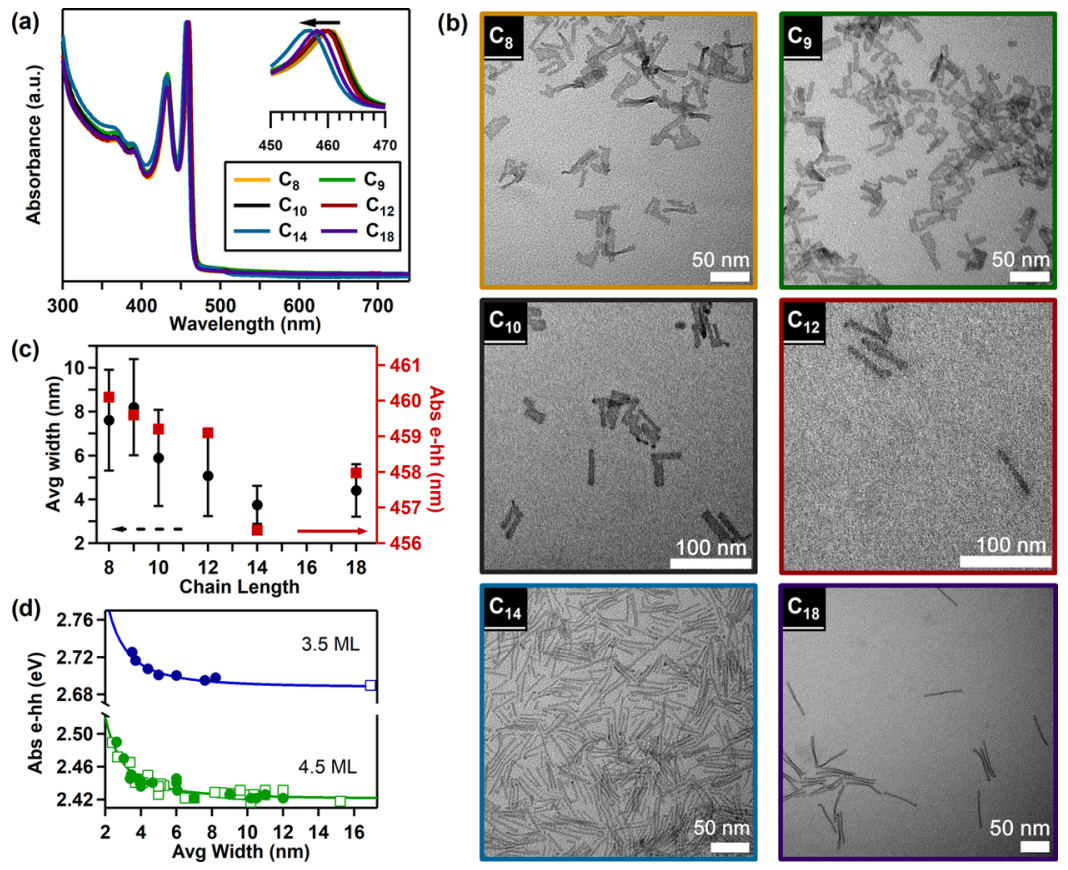

Figure 2. (a) Absorbance spectra of the purified NPLs synthesized according to procedure (1), using different cadmium carboxylates. (b) TEM images of the corresponding NPLs. (c) Comparison of the NPL width (left axis, black dots) and the spectral position of the e-hh transition (right axis, red squares), plotted against the chain length of the cadmium carboxylate. The error bars indicate the size dispersion of the NPL ensemble. (d) Sizing curves for the 3.5 ML (blue line) and 4.5 ML (green line) NPLs. For both thicknesses, filled circles represent our experimental data, while the empty squares refer to literature reports. ${ }^{9,13,16,18,27,28,34-39}$

Table 1. Width and Length (Including in Brackets the Dispersion Across the NPL Ensemble), Lateral Aspect Ratio (AR), NPL Area, Spectral Position $\lambda_{\text {e-hh }}$ and Full Width at Half-Maximum of the First Absorption Peak, PL Peak Position $\lambda_{\text {PL }}$ and Full Width at Half-Maximum, Band-Edge PL QE, and Amplitude-Weighted Average Lifetime $\tau_{\text {avg }}$ of 3.5 ML NPLs Synthesized with Different Chain Lengths (CL) According to Procedure (1)

$\begin{array}{lcccccccccc}\mathrm{CL} & \text { width }(\mathrm{nm}) & \text { length }(\mathrm{nm}) & \mathrm{AR} & \text { area }\left(\mathrm{nm}^{2}\right) & \lambda_{\text {e-hh }}(\mathrm{nm}) & \mathrm{FWHM}_{\text {e-hh }}(\mathrm{nm}) & \lambda_{\mathrm{PL}}(\mathrm{nm}) & \mathrm{FWHM}_{\mathrm{PL}}(\mathrm{nm}) & \mathrm{QE}(\%) & \tau_{\text {avg }}(\mathrm{ns}) \\ \mathrm{C}_{8} & 7.6(2) & 36.5(12) & 4.4 & 277 & 460 & 12 & 463 & 8 & 5 \\ \mathrm{C}_{9} & 8.0(2) & 24.2(10) & 3.0 & 199 & 460 & 14 & 462 & 8 & 6 \\ \mathrm{C}_{10} & 6.0(2) & 32.3(8) & 5.5 & 190 & 459 & 12 & 462 & 8 & 4.7 \\ \mathrm{C}_{12} & 5.0(2) & 30.2(10) & 6.0 & 153 & 459 & 12 & 461 & 8 & 5 \\ \mathrm{C}_{14} & 3.7(1) & 39.9(12) & 10.7 & 149 & 457 & 12 & 459 & 8 & 20 \\ \mathrm{C}_{18} & 4.4(1) & 67.5(8) & 15.3 & 297 & 458 & 12 & 460 & 8.1 & 30 \\ \end{array}$

spectra for the QD background absorption (SI, Figure S4). We considered the final aliquot (20 min for our dataset, $45 \mathrm{~min}$ for the reference procedure ${ }^{18}$ ) of each synthesis using different carboxylate precursors. To extract the absorbance at $309 \mathrm{~nm}$ originating from the NPLs, we rescaled the absorbance spectrum of the purified NPL suspension with the amplitude of the first absorbance peak, as obtained from the aliquot (SI, Figure S5). Hence, as all aliquots are taken with the same volume, the rescaled absorbance at $309 \mathrm{~nm}$ of the purified NPL suspension allows us to calculate the NPL yield via the intrinsic absorption coefficient of 3.5 ML NPLs reported by Achtstein et al. ${ }^{9}$ The yield (Figure 1c, blue squares) is calculated against the amount of Se used. All values considered in the calculation, together with their spectral positions, are summarized in Table $\mathrm{S} 1$. As suggested by the increasing $\mathrm{QD}$ side product formation, a significantly larger yield is obtained for $\mathrm{C}_{8}$ and $\mathrm{C}_{9}$ ligands compared to the reference procedure, and it decreases with increasing chain length (Figure 1c, blue squares), to a point where use of $\mathrm{C}_{18}$ ligands results in a $35 \%$ lower yield compared to literature. $^{18}$
On the other hand, the chain length of the initial carboxylate remains key to regulate the NPL growth. In Figure 2a, we show the normalized absorbance spectra of the NPLs obtained using different cadmium carboxylate precursors using procedure (1) with a growth time of $20 \mathrm{~min}$, displaying a progressive blue shift for longer chain lengths. As the NPL thickness is constant, the spectral shift of the band-edge transition is due to increased lateral quantum confinement, as confirmed by the correlation of the e-hh transition with the NPL width, obtained from transmission electron microscopy (TEM, Figure $2 \mathrm{~b}$ and Table 1). To appreciate the lateral size dispersion and associated spectral line widths, TEM histogram data and the FWHM of the first absorption peak are reported in Table 1 and Figure S6. In Figure 2c, we plot the e-hh transition for the different purified NPLs. For $\mathrm{C}_{12}, \mathrm{C}_{14}$, and $\mathrm{C}_{18}$ precursors, the average width falls below the bulk exciton Bohr radius for zincblende CdSe $(5.4 \mathrm{~nm}),{ }^{33}$ demonstrating that we are able to synthesize 3.5 ML NPLs with significant lateral confinement. TEM data further reveal that using a longer chain length, a higher lateral aspect ratio is obtained (Table 1). Our results thus support the hypothesis that longer ligands lead to an enhanced packing 
order on the NPL surface, including the side facets. ${ }^{20,22}$ We speculate that, once attached to the larger edge, they form a more ordered and thus compact ligand shell, further suppressing growth along this direction in favor of the smaller edge, thereby promoting a more pronounced anisotropic growth. Note that the reduced sizes also leads to improved colloidal stability of our samples, as NPL suspensions prepared using the current method remained dispersed in hexane for over 1 year (SI, Figure S7).

Due to the high aspect ratio, the width can be regarded as the confinement-inducing dimension. As a result, we can determine a sizing curve, correlating the spectral position of the first absorption peak to the NPL width (Figure $2 \mathrm{~d}$, blue data). A fit to the data yields the following expression for 3.5 ML NPLs

$$
E_{\mathrm{g}}(\mathrm{eV})=2.69+0.40 / w^{2}
$$

As the thickness is constant, the trend reflects the influence of the additional confinement along the width direction $(w$, in $\mathrm{nm}$ ). Interestingly, in contrast to the scaling of the band gap with thickness $d$, which follows a $1 / d$ dependence ${ }^{13}$ for $d=$ $1.0-2.9 \mathrm{~nm}$ thick NPLs, here, we obtain a $1 / w^{2}$ dependence, likely because the width remains more comparable to the Bohr radius (Table 1). The good agreement between experimental data and fit sets eq 2 as a convenient instrument to assess the width of thin NPLs using absorbance spectra. Moreover, we extended the fit to $4.5 \mathrm{ML}$ NPLs (Figure 2c, green data), collecting data from our experiments, where 4.5 ML NPLs were synthesized by slight adaptation of reported procedures $^{3,23}$ and from literature results. $9,13,16,27,28,34-39$ We obtained a similar evolution

$$
E_{\mathrm{g}}(\mathrm{eV})=2.42+0.40 / w^{2}
$$

The two fits yield the same scaling factor, which can be expected as long as materials parameters such as the effective masses of electrons and holes or dielectric constant do not vary strongly with thickness. The first constant, $E_{\infty}(n \mathrm{ML})$, can be regarded, for a specific thickness, as the band gap of an NPL with infinite width. Practically, this implies that we can combine both the confinement in thickness and width into the following expression $\left(E_{0}=1.49 \mathrm{eV},{ }^{13} d\right.$ and $w$ in $\left.\mathrm{nm}\right)$

$$
E_{\mathrm{g}}(\mathrm{eV})=E_{0}+1.27 / d+0.40 / w^{2}
$$

The emission properties of the NPLs synthesized according to eq 1 are summarized in Figure 3 and Table 1. All NPLs present a narrow band-edge emission with an FWHM of $8 \mathrm{~nm}$ and a small Stokes shift of 2-4 nm (Figure 3a and Table 1). As expected, the trend observed in absorption is translated into the PL spectra. A reduction in width, from $\mathrm{C}_{8}$ to $\mathrm{C}_{18}$, results in a spectral blue shift of the emission peak, from $464 \mathrm{~nm}$ for $\mathrm{C}_{8}$ to $460 \mathrm{~nm}$ for $\mathrm{C}_{18}$. Together with the band-edge emission, a broad band of reduced intensity can be discerned in the spectra. As already discussed for 4.5 ML and 5.5 ML, ${ }^{15,40}$ an incomplete passivation of dangling bonds at side facets reduces the PL QE, with Se-rich sites responsible for deep-band hole trapping. ${ }^{15}$ Reducing the NPL thickness to $3.5 \mathrm{ML}$, trapping at the edges and vertices is likely further amplified, inducing the presence of a small but measurable broad trap band. Nevertheless, the reduced area of our NPLs, combined with the higher synthesis temperature, leads to band-edge PL QEs in the range of 5-30\%, significantly higher than the value for our reference sample ${ }^{18}$ (PL QE of about 1\%, SI, Figure S8).
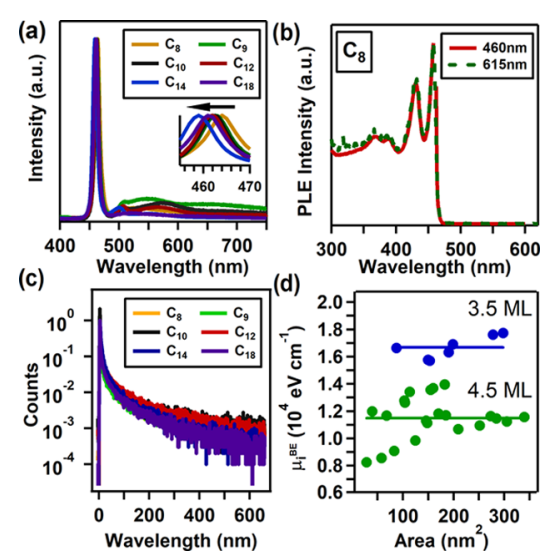

Figure 3. Emission properties of the NPLs obtained by procedure (1) using different chain lengths: (a) PL spectra, (b) PLE spectra with $\lambda_{\mathrm{em}}$ at the band edge and at $615 \mathrm{~nm}$, and (c) PL decay traces for the NPLs synthesized with $\mathrm{C}_{8}$. (d) Band-edge intrinsic absorption coefficients versus NPL area for 3.5 ML (blue) and 4.5 ML (green) NPLs.

The broad band can be attributed to trap emission, as confirmed by the photoluminescence excitation (PLE) spectra, measured at the emission wavelength of the band edge and at $615 \mathrm{~nm}$ (Figure 3b and SI, Figure S9). Notably, the PLE spectra also reveal that the two higher-energy peaks observed below $400 \mathrm{~nm}$ in the absorbance spectra (Figures 2a and $3 \mathrm{~b}$ ) pertain to the 3.5 ML NPLs. As we now also have access to size-controlled NPLs, further support was found by the correlation of the spectral position of these peaks with that of the first absorption peak (SI, Figure S10).

The amplitude-weighted average lifetime of the band-edge emission falls in the range of 3-8 ns. Curve fitting and related parameters of the PL decay traces are reported in Figure S11 and Table S2. Our values are slightly faster than 4.5 ML (5.3 $\mathrm{ns})$ to $8.5 \mathrm{ML}(10.8 \mathrm{~ns}) \mathrm{NPLs}$, demonstrating that blueemitting NPLs have a larger radiative recombination rate than the thicker green- and red-emitting CdSe NPLs (Figure 3c).,13 Nevertheless, the presence of trap emission obscures a more detailed comparison. We therefore also compared the NPL absorption coefficients, obtained by normalizing absorbance spectra at $309 \mathrm{~nm}$ to the absorption coefficients of Achtstein et al. ${ }^{9}$ Figure $3 \mathrm{~d}$ shows that the absorption coefficient of $3.5 \mathrm{ML}$ NPLs $\left(16.7 \pm 0.810^{3} \mathrm{eV} \mathrm{cm}^{-1}\right)$ is on average 1.5 times larger than 4.5 ML NPLs $\left(11.5 \pm 1.610^{3} \mathrm{eV} \mathrm{cm}^{-1}\right)$. Considering a similar local field factor for both thicknesses and correcting for the different thickness yields a band-edge oscillator strength ${ }^{41}$ that is about 1.2 times higher in 3.5 ML NPLs. As the oscillator strength for $2 \mathrm{D}$ excitons scales with the inverse of the exciton area $^{42}$ data strengthen the recent observation of a reduced exciton Bohr radius in thinner NPLs. Brumberg et al. calculated, depending on lateral size, a reduction of $11-22 \%$ in the exciton area. ${ }^{43}$ This is in agreement with the $20 \%$ increase in oscillator strength observed here. From the absorption coefficient, when also correcting for the different emission wavelengths of 3.5 ML (462 mn) and 4.5 ML (512 $\mathrm{nm})$ NPLs, we calculate a radiative recombination rate that is about 1.8 times faster. Considering that slow trap band emission influences the obtained effective decay constants for 3.5 ML NPLs, we obtain a reasonable agreement with results from the PL decay traces, where also a slight enhancement was observed. Hence, reducing the NPL thickness provides a viable pathway to obtaining faster and stronger NPL absorbers. At 
the same time, all 3.5 and 4.5 ML NPLs show similar absorption coefficients, independent of lateral area, confirming earlier results that the so-called giant oscillator strength effect, i.e., an oscillator strength that scales with the NPL area, only applies at cryogenic temperatures, ${ }^{44,45}$ and is canceled at room temperature by phonon and/or defect scattering. ${ }^{46}$ This is also supported by comparing the NPL absorption coefficient with those of OD QDs that have a band gap absorption at similar wavelengths. Using previously published data of Capek et al. ${ }^{47}$ and Jasieniak et al. ${ }^{48}$ (Figure S12), we obtain values of 15.8 and $10.1 \mathrm{~cm}^{-1} \mathrm{eV}$ at 462 and $512 \mathrm{~nm}$, respectively, values that are not substantially different from their $2 \mathrm{D}$ counterparts.

Finally, as we have developed a procedure for the synthesis of 3.5 ML NPLs at a growth temperature of $220^{\circ} \mathrm{C}$, we can now again investigate the NPL growth at reduced temperature to explore if thinner NPLs can be obtained. The synthesis procedure (1) was therefore conducted at $160{ }^{\circ} \mathrm{C}$. The main obstacle for a direct extension of the procedure (1) to lower temperature is the reduced solubility of Se-mesh. For this reason, we slightly adapted our procedure, injecting soluble TOP-Se together with propionic acid at $160{ }^{\circ} \mathrm{C}$, in accordance with recent reports on $2.5 \mathrm{ML}$ NPLs. ${ }^{17,19}$

The results are shown in Figure 4. Following the lower growth temperature, we were able to isolate NPLs with a band-

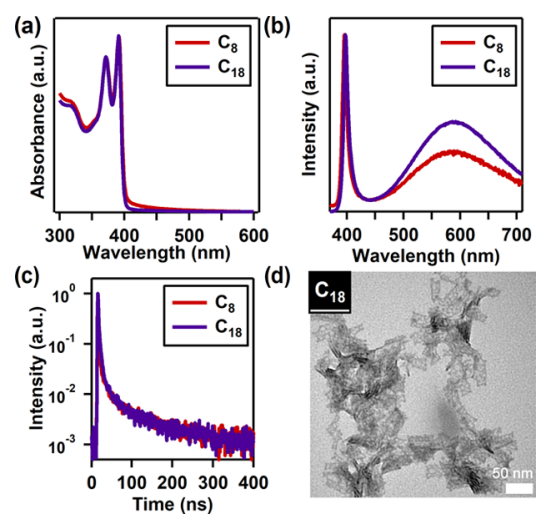

Figure 4. NPL optical properties produced by a modified version of procedure (1), using $\mathrm{C}_{8}$ and $\mathrm{C}_{18}$ cadmium carboxylates. (a) Absorbance spectra, (b) PL spectra, (c) PL decay traces, and (d) TEM image of the $2.5 \mathrm{ML}$ CdSe synthesized with cadmium stearate $\left(C_{18}\right)$. The electron beam induced some holes in the $0.76 \mathrm{~nm}$ NPLs.

edge absorption at $393 \mathrm{~nm}$, consistent with $2.5 \mathrm{ML}$ CdSe NPLs (Figure 4a). Again, the reaction could be conducted with different chain lengths for the metal precursor, here demonstrated using $\mathrm{C}_{8}$ as well as $\mathrm{C}_{18}$. As for the luminescent properties, a narrow UV band-edge emission, with an FWHM of about $10 \mathrm{~nm}$, is observed at $397 \mathrm{~nm}$ (Figure $4 \mathrm{~b}$ and Table 2). This is again accompanied by a broad trap band, which has a larger amplitude than the 3.5 ML NPLs, yet is lower than typical reported spectra. ${ }^{3,17,19}$ Despite the strongly reduced

Table 2. PL Peak Position $\lambda_{\text {PL }}$, Full Width at HalfMaximum, Band-Edge PL QE, and Amplitude-Weighted Average Lifetime $\tau_{\text {avg }}$ of 2.5 ML CdSe NPLs Synthesized with Different Precursor Chain Lengths (CL)

$\begin{array}{lcccc}\mathrm{CL} & \lambda_{\mathrm{PL}}(\mathrm{nm}) & \mathrm{FWHM}_{\mathrm{PL}}(\mathrm{nm}) & \mathrm{QE}(\%) & \tau_{\mathrm{avg}}(\mathrm{ns}) \\ \mathrm{C}_{8} & 396 & 8 & 5 & 2.6 \\ \mathrm{C}_{18} & 397 & 8 & 11 & 3.0\end{array}$

thickness, we obtained appreciable band-edge PL QEs of 5\% $\left(\mathrm{C}_{8}\right)$ and $11 \%\left(\mathrm{C}_{18}\right)$. Literature data to compare with, to the best of our knowledge, are only provided by a recent paper of Delikanli et al., who report an overall (i.e., band-edge and trap band) PL QE below 20\%, where one has to consider that the band-edge emission only constitutes a small fraction of the total emission spectrum. ${ }^{17}$ As for 3.5 ML NPLs, the temporal evolution of the reaction yields a progressive red shift of the ehh transition, from $388 \mathrm{~nm}$ at $2 \mathrm{~min}$ to $393 \mathrm{~nm}$ at $20 \mathrm{~min}$ (Figure S13); note however that due to the lower temperature used, we no longer obtain NPLs with strong lateral confinement at longer reaction time. Time-resolved fluorescence decay measurements (Figures $4 \mathrm{c}$ and S14, and Table S3) yielded average decay times of $2.6 \mathrm{~ns}\left(\mathrm{C}_{8}\right)$ and $3.0 \mathrm{~ns}\left(\mathrm{C}_{18}\right)$. In line with 3.5 ML NPLs, we observed a further reduction of the recombination rate, opening up prospects for ultrafast blue and UV emitters based on 2D colloidal nanocrystals. While it is difficult to size the NPLs by TEM analysis (Figure 4d), as sample damage occurs when they are merely $0.76 \mathrm{~nm}$ thick, we clearly demonstrated that the synthesis method can be extended to obtain fluorescent 2.5 ML CdSe NPLs.

\section{CONCLUSIONS}

We showed the possibility to tune the width and aspect ratio of 3.5 ML CdSe NPLs, using cadmium carboxylate precursors with different chain lengths. We observed a dependence of the chemical yield of the reaction on the length of the cadmium carboxylate, with shorter ligands having higher yields due to a suppression of QD side product formation. The different ligands, presumably due to the different packing order, enable us to control the width and the aspect ratio of the resulting NPLs. This allowed us to finely tune their lateral dimensions, without resorting to shorter growth times or reduced temperatures. The synthesis method yields NPLs with a narrow band-edge emission, a band-edge PL QE up to $30 \%$, and fast emission lifetime down to $3 \mathrm{~ns}$. By slightly adjusting the synthesis protocol, we can also obtain 2.5 ML CdSe NPLs with distinct band-edge emission, PL QE up to $11 \%$, and emission lifetime down to $2.6 \mathrm{~ns}$, demonstrating the generality of our approach. Establishing a reproducible and tunable preparation method of thin CdSe NPL cores now allows for further in-depth fundamental studies on their size- and thickness-dependent photophysics, and further work on the growth of heterostructured blue and UV NPLs offers interesting prospects for efficient UV or blue/green light sources to cover applications such as light-emitting diodes (LEDs) or lasers.

\section{EXPERIMENTAL SECTION}

Materials. Cadmium nitrate tetrahydrate (99.997\%), sodium myristate $(\geq 99 \%)$, cadmium oxide (CdO, $\geq 99.99 \%)$, stearic acid $(\geq 97.0 \%)$, myristic acid $(\geq 98.0 \%)$, octanoic acid $(\geq 98.0 \%)$, decanoic acid $(\geq 98.0 \%)$, and dodecanoic acid $(98.0 \%)$ were purchased from Sigma-Aldrich. Nonanoic acid (97.0\%), 1-octadecene (ODE, 90\%), and oleic acid (OA, 90\%) were purchased from Alfa Aesar. Propionic acid (>99.5\%), hexane (>99\%), Disolol® (ethanol, EtOH, 98\%), and 2-propanol (IPA, >99\%) were purchased from Chem Lab. Selenium powder $(\geq 99.99 \%)$ was purchased from Acros Organics. All chemicals were used without further purification.

Cadmium Carboxylate $\left(\mathrm{Cd}(\mathrm{OOCR})_{2}\right)$ Preparation. All cadmium carboxylates were prepared starting from CdO. In a three-neck flask, $2 \mathrm{~g}(15.7 \mathrm{mmol})$ of $\mathrm{CdO}$ was added to $40 \mathrm{mmol}$ of the respective carboxylic acid. Under stirring, the system was heated under $\mathrm{N}_{2}$ atmosphere to $210{ }^{\circ} \mathrm{C}$, until a colorless solution was 
obtained. The reaction was kept at $210{ }^{\circ} \mathrm{C}$ for $20 \mathrm{~min}$ and then cooled. During cooling, between 110 and $80{ }^{\circ} \mathrm{C}$, the mixture was connected to a vacuum line to remove water produced in the condensation reaction. The colorless solution was rapidly transferred to centrifugation tubes, acetone was added, and a white solid precipitated after centrifugation. The solid fraction was centrifuged with fresh acetone three additional times to purify the Cd(OOCR $)_{2}$ reaction product, and then dried overnight under vacuum. To validate this synthesis, cadmium myristate was also prepared according to a procedure previously reported in the literature. ${ }^{18}$ No appreciable differences in NPL synthesis were observed using the two different myristate precursors.

Synthesis of 3.5 ML CdSe NPLs with Different Cadmium Carboxylates. In a $25 \mathrm{~mL}$ three-neck round-bottom flask, $0.50 \mathrm{mmol}$ of $\mathrm{Cd}(\mathrm{OOCR})_{2}\left(\mathrm{R}=-\mathrm{C}_{7} \mathrm{H}_{15},-\mathrm{C}_{8} \mathrm{H}_{17},-\mathrm{C}_{9} \mathrm{H}_{19},-\mathrm{C}_{11} \mathrm{H}_{23}-\mathrm{C}_{13} \mathrm{H}_{27}\right.$, $\left.-\mathrm{C}_{17} \mathrm{H}_{35}\right)$ and $0.26 \mathrm{mmol}$ of Se powder were mixed with $12 \mathrm{~mL}$ of ODE. The suspension was degassed for $1 \mathrm{~h}$ at $100{ }^{\circ} \mathrm{C}$, followed by heating the mixture, under $\mathrm{N}_{2}$, to $160^{\circ} \mathrm{C}$ for $10 \mathrm{~min}$. The temperature was then set to $220{ }^{\circ} \mathrm{C}$, and, at $200{ }^{\circ} \mathrm{C}, 100 \mu \mathrm{L}(1.33 \mathrm{mmol})$ of propionic acid dispersed in $1 \mathrm{~mL}$ of ODE was swiftly injected into the flask. The reaction proceeded for $20 \mathrm{~min}$ at $220^{\circ} \mathrm{C}$, then heating was removed to cool down the system. When the temperature reached $160{ }^{\circ} \mathrm{C}, 2 \mathrm{~mL}$ of $\mathrm{OA}$ was injected and the suspension was cooled to $80{ }^{\circ} \mathrm{C}$, to proceed to purification.

Synthesis of 2.5 ML CdSe NPLs with Different Cadmium Carboxylates. In a $25 \mathrm{~mL}$ three-neck round-bottom flask, $0.50 \mathrm{mmol}$ of $\mathrm{Cd}(\mathrm{OOCR})_{2}\left(\mathrm{R}=-\mathrm{C}_{7} \mathrm{H}_{15},-\mathrm{C}_{17} \mathrm{H}_{35}\right)$ and $12 \mathrm{~mL}$ of ODE were degassed for $1 \mathrm{~h}$ at $100{ }^{\circ} \mathrm{C}$. The temperature was set to $160{ }^{\circ} \mathrm{C}$, and, at $150{ }^{\circ} \mathrm{C}, 100 \mu \mathrm{L}(1.33 \mathrm{mmol})$ of propionic acid dispersed in $1 \mathrm{~mL}$ of ODE and $100 \mu \mathrm{L}$ of a $1 \mathrm{M}$ TOP-Se solution were swiftly injected into the flask. The reaction proceeded for $20 \mathrm{~min}$ at $160{ }^{\circ} \mathrm{C}$, then heating was removed, and $2 \mathrm{~mL}$ of $\mathrm{OA}$ was added. The suspension was cooled to $80{ }^{\circ} \mathrm{C}$ to proceed to purification.

Synthesis of 4.5 ML CdSe NPLs with Different Lateral Dimensions. The synthesis of 4.5 ML NPLs with different aspect ratios was slightly adapted from reported procedures. ${ }^{3,23}$ Briefly, in a $25 \mathrm{~mL}$ three-neck round-bottom flask, $0.30 \mathrm{mmol}$ of $\mathrm{Cd}$ (myristate $)_{2}$ and $0.30 \mathrm{mmol}$ of Se powder were mixed with $12 \mathrm{~mL}$ of ODE. The suspension was degassed for $1 \mathrm{~h}$ at $100{ }^{\circ} \mathrm{C}$, then, under $\mathrm{N}_{2}$ atmosphere, the temperature was set to $240{ }^{\circ} \mathrm{C}$, and when the suspension became deep orange (at $215-220{ }^{\circ} \mathrm{C}$ ), $0.6 \mathrm{mmol}$ of $\mathrm{Cd}(\mathrm{ac})_{2}$ or $\mathrm{Cd}(\mathrm{Ac})_{2} \cdot 2 \mathrm{H}_{2} \mathrm{O}$ was swiftly injected. The reaction temperature was set at $240{ }^{\circ} \mathrm{C}$, and the time was varied from $45 \mathrm{~s}$ to $10 \mathrm{~min}$ to tune the lateral dimensions. Afterward, the suspension was cooled, and when the temperature reached $160^{\circ} \mathrm{C}, 2 \mathrm{~mL}$ of OA was injected, followed by cooling to $80^{\circ} \mathrm{C}$, to proceed to purification.

Purification Protocol for CdSe Nanoplatelets (NPLs). The crude synthesis product was mixed with $20 \mathrm{~mL}$ of $n$-hexane, and 15 $\mathrm{mL}$ of a $1: 5(\mathrm{v} / \mathrm{v})$ solution of IPA/EtOH was added. The suspension was centrifuged for $10 \mathrm{~min}$ at $4300 \mathrm{rpm}$. The NPLs precipitated under these conditions, while the byproducts (dots, clusters) remained in the liquid phase. The supernatant was discarded, and the solid was redispersed in $n$-hexane and centrifuged at $4200 \mathrm{rpm}$ to remove the unreacted carboxylates. The solid was discarded, and the supernatant, containing CdSe NPLs, was collected and stored as $n$-hexane dispersion.

Synthesis Yield Determination. To follow the evolution of the reactions, aliquots were collected at specific times, before (160 and $\left.200{ }^{\circ} \mathrm{C}\right)$ and after $(120,300,600,1200 \mathrm{~s})$ the injection of propionic acid. With a syringe, $200 \mu \mathrm{L}$ of the mixture was rapidly transferred into a vial containing $50 \mu \mathrm{L}$ of OA. Depending on the sample, 25-35 $\mu \mathrm{L}$ of this suspension was then added to $3 \mathrm{~mL}$ of hexane, and this diluted sample was used to measure the absorbance spectra of the related aliquot. The dilution for each sample is reported in the SI (Table S1).

Transmission Electron Microscopy. Samples were prepared by drop-casting dilute $n$-hexane dispersions onto carbon-coated copper grids. Bright-field TEM images were acquired on a JEOL JEM-1011 microscope (W filament) operating at an accelerating voltage of 60 $\mathrm{kV}$.
Optical Characterization. Absorbance spectra were taken with a PerkinElmer Lambda 950 spectrometer. Photoluminescence measurements were performed on an Edinburgh Instruments FLSP920 UVvis-NIR spectrofluorometer, using a $450 \mathrm{~W}$ xenon lamp as the excitation source for PL, PLE, and PL QE measurements. An excitation wavelength of $380 \mathrm{~nm}$ was used for all steady-state emission spectra. The PL QE was measured using an integrating sphere. The PL decay traces were recorded by exciting the samples at $331 \mathrm{~nm}$ using an Edinburgh EPLED-330 (pulse width: 871.7 ps) at a repetition rate of $0.5-1 \mathrm{MHz}$.

\section{ASSOCIATED CONTENT}

\section{Supporting Information}

The Supporting Information is available free of charge at https://pubs.acs.org/doi/10.1021/acs.chemmater.0c03066.

Additional absorbance, steady-state and time-resolved PL spectra, tables with PL decay fit constants, TEM images, and histograms (PDF)

\section{AUTHOR INFORMATION}

\section{Corresponding Author}

Iwan Moreels - Department of Chemistry, Ghent University, 9000 Ghent, Belgium; 이이이.org/0000-0003-3998-7618; Email: iwan.moreels@ugent.be

\section{Authors}

Alessio Di Giacomo - Department of Chemistry, Ghent University, 9000 Ghent, Belgium

Carmelita Rodà - Department of Chemistry, Ghent University, 9000 Ghent, Belgium

Ali Hossain Khan - Department of Chemistry, Ghent University, 9000 Ghent, Belgium; (1) orcid.org/0000-00017155-0200

Complete contact information is available at:

https://pubs.acs.org/10.1021/acs.chemmater.0c03066

\section{Author Contributions}

The manuscript was written through contributions of all authors. All authors have given approval to the final version of the manuscript.

\section{Funding}

This project has received funding from the European Research Council (ERC) under the European Union's Horizon 2020 research and innovation program (grant agreement no. 714876 PHOCONA).

\section{Notes}

The authors declare no competing financial interest.

\section{ACKNOWLEDGMENTS}

The authors acknowledge Katrien Haustraete, and the TEM facility of the Nematology Research Unit, member of the UGent TEM Expertise center (Life Sciences) for assistance with TEM imaging, and Matteo Di Giacomo for assistance with creating the Table of Contents figure.

\section{REFERENCES}

(1) Joo, J.; Son, J. S.; Kwon, S. G.; Yu, J. H.; Hyeon, T. Lowtemperature solution-phase synthesis of quantum well structured CdSe nanoribbons. J. Am. Chem. Soc. 2006, 128, 5632-5633.

(2) Ithurria, S.; Dubertret, B. Quasi 2D colloidal CdSe platelets with thicknesses controlled at the atomic level. J. Am. Chem. Soc. 2008, 130, 16504-16505. 
(3) Ithurria, S.; Tessier, M. D.; Mahler, B.; Lobo, R. P.; Dubertret, B.; Efros, A. L. Colloidal nanoplatelets with two-dimensional electronic structure. Nat. Mater. 2011, 10, 936-941.

(4) Novoselov, K. S.; Geim, A. K.; Morozov, S. V.; Jiang, D.; Zhang, Y.; Dubonos, S. V.; Grigorieva, I. V.; Firsov, A. A. Electric field effect in atomically thin carbon films. Science 2004, 306, 666-669.

(5) Tessier, M. D.; Javaux, C.; Maksimovic, I.; Loriette, V.; Dubertret, B. Spectroscopy of single CdSe nanoplatelets. ACS Nano 2012, 6, 6751-6758.

(6) She, C.; Fedin, I.; Dolzhnikov, D. S.; Demortiere, A.; Schaller, R. D.; Pelton, M.; Talapin, D. V. Low-threshold stimulated emission using colloidal quantum wells. Nano Lett. 2014, 14, 2772-2777.

(7) Diroll, B. T.; Talapin, D. V.; Schaller, R. D. Violet-to-Blue Gain and Lasing from Colloidal CdS Nanoplatelets: Low-Threshold Stimulated Emission Despite Low Photoluminescence Quantum Yield. ACS Photonics 2017, 4, 576-583.

(8) Grim, J. Q.; Christodoulou, S.; Di Stasio, F.; Krahne, R.; Cingolani, R.; Manna, L.; Moreels, I. Continuous-wave biexciton lasing at room temperature using solution-processed quantum wells. Nat. Nanotechnol. 2014, 9, 891-895.

(9) Achtstein, A. W.; Antanovich, A.; Prudnikau, A.; Scott, R.; Woggon, U.; Artemyev, M. Linear Absorption in CdSe Nanoplates: Thickness and Lateral Size Dependency of the Intrinsic Absorption. J. Phys. Chem. C 2015, 119, 20156-20161.

(10) Giovanella, U.; Pasini, M.; Lorenzon, M.; Galeotti, F.; Lucchi, C.; Meinardi, F.; Luzzati, S.; Dubertret, B.; Brovelli, S. Efficient Solution-Processed Nanoplatelet-Based Light-Emitting Diodes with High Operational Stability in Air. Nano Lett. 2018, 18, 3441-3448.

(11) Chen, Z.; Nadal, B.; Mahler, B.; Aubin, H.; Dubertret, B. Quasi2D Colloidal Semiconductor Nanoplatelets for Narrow Electroluminescence. Adv. Funct. Mater. 2014, 24, 295-302.

(12) Lorenzon, M.; Christodoulou, S.; Vaccaro, G.; Pedrini, J.; Meinardi, F.; Moreels, I.; Brovelli, S. Reversed oxygen sensing using colloidal quantum wells towards highly emissive photoresponsive varnishes. Nat. Commun. 2015, 6, No. 6434.

(13) Christodoulou, S.; Climente, J. I.; Planelles, J.; Brescia, R.; Prato, M.; Martin-Garcia, B.; Khan, A. H.; Moreels, I. ChlorideInduced Thickness Control in CdSe Nanoplatelets. Nano Lett. 2018, $18,6248-6254$.

(14) Cho, W.; Kim, S.; Coropceanu, I.; Srivastava, V.; Diroll, B. T.; Hazarika, A.; Fedin, I.; Galli, G.; Schaller, R. D.; Talapin, D. V. Direct Synthesis of Six-Monolayer (1.9 nm) Thick Zinc-Blende CdSe Nanoplatelets Emitting at $585 \mathrm{~nm}$. Chem. Mater. 2018, 30, 69576960.

(15) Singh, S.; Tomar, R.; Ten Brinck, S.; De Roo, J.; Geiregat, P.; Martins, J. C.; Infante, I.; Hens, Z. Colloidal CdSe Nanoplatelets, A Model for Surface Chemistry/Optoelectronic Property Relations in Semiconductor Nanocrystals. J. Am. Chem. Soc. 2018, 140, 1329213300 .

(16) Meerbach, C.; Wu, C.; Erwin, S. C.; Dang, Z.; Prudnikau, A.; Lesnyak, V. Halide-Assisted Synthesis of Cadmium Chalcogenide Nanoplatelets. Chem. Mater. 2020, 32, 566-574.

(17) Delikanli, S.; Yu, G.; Yeltik, A.; Bose, S.; Erdem, T.; Yu, J.; Erdem, O.; Sharma, M.; Sharma, V. K.; Quliyeva, U.; Shendre, S.; Dang, C.; Zhang, D. H.; Sum, T. C.; Fan, W.; Demir, H. V. Ultrathin Highly Luminescent Two-Monolayer Colloidal CdSe Nanoplatelets. Adv. Funct. Mater. 2019, 29, No. 1901028.

(18) Ithurria, S.; Bousquet, G.; Dubertret, B. Continuous transition from $3 \mathrm{D}$ to $1 \mathrm{D}$ confinement observed during the formation of CdSe nanoplatelets. J. Am. Chem. Soc. 2011, 133, 3070-3077.

(19) Kurtina, D. A.; Garshev, A. V.; Vasil'eva, I. S.; Shubin, V. V.; Gaskov, A. M.; Vasiliev, R. B. Atomically Thin Population of Colloidal CdSe Nanoplatelets: Growth of Rolled-up Nanosheets and Strong Circular Dichroism Induced by Ligand Exchange. Chem. Mater. 2019, 31, 9652-9663.

(20) Jiang, Y.; Ojo, W. S.; Mahler, B.; Xu, X.; Abecassis, B.; Dubertret, B. Synthesis of CdSe Nanoplatelets without Short-Chain Ligands: Implication for Their Growth Mechanisms. ACS Omega 2018, 3, 6199-6205.
(21) Sharma, M.; Gungor, K.; Yeltik, A.; Olutas, M.; Guzelturk, B.; Kelestemur, Y.; Erdem, T.; Delikanli, S.; McBride, J. R.; Demir, H. V. Near-Unity Emitting Copper-Doped Colloidal Semiconductor Quantum Wells for Luminescent Solar Concentrators. Adv. Mater. 2017, 29, No. 1700821.

(22) Li, Z.; Peng, X. Size/shape-controlled synthesis of colloidal CdSe quantum disks: ligand and temperature effects. J. Am. Chem. Soc. 2011, 133, 6578-6586.

(23) Bertrand, G. H.; Polovitsyn, A.; Christodoulou, S.; Khan, A. H.; Moreels, I. Shape control of zincblende CdSe nanoplatelets. Chem. Commun. 2016, 52, 11975-11978.

(24) Sugimoto, T. Preparation of monodispersed colloidal particles. Adv. Colloid Interface Sci. 1987, 28, 65-108.

(25) Kwon, S. G.; Piao, Y.; Park, J.; Angappane, S.; Jo, Y.; Hwang, N. M.; Park, J. G.; Hyeon, T. Kinetics of monodisperse iron oxide nanocrystal formation by "heating-up" process. J. Am. Chem. Soc. 2007, 129, 12571-12584.

(26) Hens, Z.; Čapek, R. K. Size tuning at full yield in the synthesis of colloidal semiconductor nanocrystals, reaction simulations and experimental verification. Coord. Chem. Rev. 2014, 263-264, 217228.

(27) She, C.; Fedin, I.; Dolzhnikov, D. S.; Dahlberg, P. D.; Engel, G. S.; Schaller, R. D.; Talapin, D. V. Red, Yellow, Green, and Blue Amplified Spontaneous Emission and Lasing Using Colloidal CdSe Nanoplatelets. ACS Nano 2015, 9, 9475-9485.

(28) Tomar, R.; Kulkarni, A.; Chen, K.; Singh, S.; van Thourhout, D.; Hodgkiss, J. M.; Siebbeles, L. D. A.; Hens, Z.; Geiregat, P. Charge Carrier Cooling Bottleneck Opens Up Nonexcitonic Gain Mechanisms in Colloidal CdSe Quantum Wells. J. Phys. Chem. C 2019, 123, $9640-9650$

(29) Castro, N.; Bouet, C.; Ithurria, S.; Lequeux, N.; Constantin, D.; Levitz, P.; Pontoni, D.; Abecassis, B. Insights into the Formation Mechanism of CdSe Nanoplatelets Using in Situ X-ray Scattering. Nano Lett. 2019, 19, 6466-6474.

(30) Knüsel, P. N.; Riedinger, A.; Rossinelli, A. A.; Ott, F. D.; Mule, A. S.; Norris, D. J. Experimental Evidence for Two-Dimensional Ostwald Ripening in Semiconductor Nanoplatelets. Chem. Mater. 2020, 32, 3312-3319.

(31) Riedinger, A.; Ott, F. D.; Mule, A.; Mazzotti, S.; Knusel, P. N.; Kress, S. J. P.; Prins, F.; Erwin, S. C.; Norris, D. J. An intrinsic growth instability in isotropic materials leads to quasi-two-dimensional nanoplatelets. Nat. Mater. 2017, 16, 743-748.

(32) Bullen, C.; van Embden, J.; Jasieniak, J.; Cosgriff, J. E.; Mulder, R. J.; Rizzardo, E.; Gu, M.; Raston, C. L. High Activity PhosphineFree Selenium Precursor Solution for Semiconductor Nanocrystal Growth. Chem. Mater. 2010, 22, 4135-4143.

(33) Norris, D. J.; Sacra, A.; Murray, C. B.; Bawendi, M. G. Measurement of the size dependent hole spectrum in CdSe quantum dots. Phys. Rev. Lett. 1994, 72, 2612-2615.

(34) Leemans, J.; Singh, S.; Li, C.; Ten Brinck, S.; Bals, S.; Infante, I.; Moreels, I.; Hens, Z. Near-Edge Ligand Stripping and Robust Radiative Exciton Recombination in CdSe/CdS Core/Crown Nanoplatelets. J. Phys. Chem. Lett. 2020, 11, 3339-3344.

(35) Geiregat, P.; Tomar, R.; Chen, K.; Singh, S.; Hodgkiss, J. M.; Hens, Z. Thermodynamic Equilibrium between Excitons and Excitonic Molecules Dictates Optical Gain in Colloidal CdSe Quantum Wells. J. Phys. Chem. Lett. 2019, 10, 3637-3644.

(36) Kelestemur, Y.; Shynkarenko, Y.; Anni, M.; Yakunin, S.; De Giorgi, M. L.; Kovalenko, M. V. Colloidal CdSe Quantum Wells with Graded Shell Composition for Low-Threshold Amplified Spontaneous Emission and Highly Efficient Electroluminescence. ACS Nano 2019, 13, 13899-13909.

(37) Castelli, A.; Dhanabalan, B.; Polovitsyn, A.; Caligiuri, V.; Di Stasio, F.; Scarpellini, A.; Brescia, R.; Palei, M.; Martín-García, B.; Prato, M.; Manna, L.; Moreels, I.; Krahne, R.; Arciniegas, M. P. Core/ Shell CdSe/CdS Bone-Shaped Nanocrystals with a Thick and Anisotropic Shell as Optical Emitters. Adv. Opt. Mater. 2020, 8, No. 1901463 
(38) Khan, A. H.; Pinchetti, V.; Tanghe, I.; Dang, Z.; Martín-García, B.; Hens, Z.; Van Thourhout, D.; Geiregat, P.; Brovelli, S.; Moreels, I. Tunable and Efficient Red to Near-Infrared Photoluminescence by Synergistic Exploitation of Core and Surface Silver Doping of CdSe Nanoplatelets. Chem. Mater. 2019, 31, 1450-1459.

(39) Halim, H.; Simon, J.; Lieberwirth, I.; Mailander, V.; Koynov, K.; Riedinger, A. Water-dispersed semiconductor nanoplatelets with high fluorescence brightness, chemical and colloidal stability. J. Mater. Chem. B 2020, 8, 146-154.

(40) Zhang, J.; Zhang, H.; Cao, W.; Pang, Z.; Li, J.; Shu, Y.; Zhu, C.; Kong, X.; Wang, L.; Peng, X. Identification of Facet-Dependent Coordination Structures of Carboxylate Ligands on CdSe Nanocrystals. J. Am. Chem. Soc. 2019, 141, 15675-15683.

(41) Moreels, I.; Lambert, K.; Smeets, D.; De Muynck, D.; Nollet, T.; Martins, J. C.; Vanhaecke, F.; Vantomme, A.; Delerue, C.; Allan, G.; Hens, Z. Size-dependent optical properties of colloidal PbS quantum dots. ACS Nano 2009, 3, 3023-3030.

(42) Feldmann, J.; Peter, G.; Gobel, E. O.; Dawson, P.; Moore, K.; Foxon, C.; Elliott, R. J. Linewidth dependence of radiative exciton lifetimes in quantum wells. Phys. Rev. Lett. 1987, 59, 2337-2340.

(43) Brumberg, A.; Harvey, S. M.; Philbin, J. P.; Diroll, B. T.; Lee,

B.; Crooker, S. A.; Wasielewski, M. R.; Rabani, E.; Schaller, R. D. Determination of the In-Plane Exciton Radius in 2D CdSe Nanoplatelets via Magneto-optical Spectroscopy. ACS Nano 2019, 13, 8589-8596.

(44) Li, Q.; Liu, Q.; Schaller, R. D.; Lian, T. Reducing the Optical Gain Threshold in Two-Dimensional CdSe Nanoplatelets by the Giant Oscillator Strength Transition Effect. J. Phys. Chem. Lett. 2019, 10, 1624-1632.

(45) Naeem, A.; Masia, F.; Christodoulou, S.; Moreels, I.; Borri, P.; Langbein, W. Giant exciton oscillator strength and radiatively limited dephasing in two-dimensional platelets. Phys. Rev. B 2015, 91, No. $121302(\mathrm{R})$.

(46) Li, Q.; Lian, T. Exciton Spatial Coherence and Optical Gain in Colloidal Two-Dimensional Cadmium Chalcogenide Nanoplatelets. Acc. Chem. Res. 2019, 52, 2684-2693.

(47) Kamal, J. S.; Omari, A.; Van Hoecke, K.; Zhao, Q.; Vantomme, A.; Vanhaecke, F.; Capek, R. K.; Hens, Z. Size-Dependent Optical Properties of Zinc Blende Cadmium Telluride Quantum Dots. J. Phys. Chem. C 2012, 116, 5049-5054.

(48) Jasieniak, J.; Smith, L.; van Embden, J.; Mulvaney, P.; Califano, M. Re-examination of the Size-Dependent Absorption Properties of CdSe Quantum Dots. J. Phys. Chem. C 2009, 113, 19468-19474. 